\title{
Comparison of mechanical circulatory support with venoarterial extracorporeal membrane oxygenation or Impella for patients with cardiogenic shock: a propensity-matched analysis
}

\author{
Konstantinos Karatolios ${ }^{1}$ (1) - Georgios Chatzis ${ }^{2} \cdot$ Birgit Markus $^{1} \cdot$ Ulrich Luesebrink $^{1} \cdot$ Holger Ahrens $^{1}$. \\ Dimitar Divchev ${ }^{1} \cdot$ Styliani Syntila ${ }^{1} \cdot$ Andreas Jerrentrup $^{2} \cdot$ Bernhard Schieffer $^{1}$
}

Received: 6 June 2020 / Accepted: 30 October 2020 / Published online: 13 November 2020

(c) The Author(s) 2020

\begin{abstract}
Background Percutaneous mechanical circulatory devices are increasingly used in patients with cardiogenic shock (CS). As evidence from randomized studies comparing these devices are lacking, optimal choice of the device type is unclear. Here we aim to compare outcomes of patients with CS supported with either Impella or vaECMO.

Methods Retrospective single-center analysis of patients with CS, from September 2014 to September 2019. Patients were assisted with either Impella 2.5/CP or vaECMO. Patients supported ultimately with both devices were analyzed according to the first device implanted. Primary outcomes were hospital and 6-month survival. Secondary endpoints were complications. Survival outcomes were compared using propensity-matched analysis to account for differences in baseline characteristics between both groups.

Results A total of 423 patients were included (Impella, $n=300$ and vaECMO, $n=123$ ). Survival rates were similar in both groups (hospital survival: Impella $47.7 \%$ and vaECMO 37.3\%, $p=0.07$; 6-month survival Impella $45.7 \%$ and vaECMO $35.8 \%, p=0.07$ ). There was no significant difference in survival rates, even after adjustment for baseline differences (hospital survival: Impella 50.6\% and vaECMO 38.6\%, $p=0.16$; 6-month survival Impella 45.8\% and vaECMO 38.6\%, $p=0.43$ ). Access-site bleeding and leg ischemia occurred more frequently in patients with vaECMO $(17 \%$ versus $7.3 \%, p=0.004$; $17 \%$ versus $7.7 \%, p=0.008$ ).

Conclusions In this retrospective analysis of patients with CS, treatment with Impella 2.5/CP or vaECMO was associated with similar hospital and 6-month survival rates. Device-related access-site vascular complications occurred more frequently in the vaECMO group. A randomized trial is warranted to examine the effects of these devices on outcomes and to determine the optimal device choice in patients with CS.
\end{abstract}

Keywords Mechanical circulatory support $\cdot$ Venoarterial extracorporeal membrane oxygenation $\cdot$ Impella $\cdot$ Cardiogenic shock

Konstantinos Karatolios and Georgios Chatzis have been contributed equally to this work.

Konstantinos Karatolios

konstantinos.karatolios@med.uni-marburg.de

1 Department of Cardiology, Angiology and Intensive Care, Philipps University Marburg, BaldingerStr., 35043 Marburg, Germany

2 Department of Emergency Medicine, Philipps University Marburg, Marburg, Germany

\section{Introduction}

Despite the increased use of evidence-based medicine and interventions, CS still portends unacceptably high hospital mortality rates. Percutaneous mechanical circulatory support (MCS) devices are increasingly used in patients with CS, in order to restore hemodynamics, improve cardiac output and ensure adequate end-organ perfusion [1]. The Impella pump and venoarterial extracorporeal membrane oxygenation (vaECMO) are the most frequently used devices for temporary percutaneous MCS in this context. However, the physiologic effects on cardiac work and hemodynamics, insertion technique as well as clinical management differ significantly 
between these two devices [2,3]. Despite the growth in the use of vaECMO and Impella to support patients with CS, no randomized trials have compared the efficacy of these devices in patients with CS. As a result, optimal selection of the device type is still a matter of debate and no specific guideline recommendation exists. Therefore, the aim of this retrospective study was to compare outcomes of patients with CS supported with either Impella (2.5/CP) or vaECMO.

\section{Methods}

\section{Study design}

We retrospectively analyzed data from all consecutive patients supported with Impella 2.5/CP or vaECMO for CS in our institution, a European tertiary University Hospital, from September 2014 to September 2019. Patients with refractory cardiac arrest in whom insertion of the device took place under ongoing cardiopulmonary resuscitation were excluded from the analysis. CS was defined as the need for continuous infusion of catecholamines to maintain systolic blood pressure $>90 \mathrm{mmHg}$ with clinical signs of pulmonary congestion and signs of end-organ hypoperfusion indicated by an elevated lactate level $>2 \mathrm{mmol} / \mathrm{L}$. Operators used MCS in patients with CS, according to our institutional practices. However, as there is insufficient evidence for the choice of the circulatory support device type in CS and neither the superiority of one device over the other was proven in the setting of CS, the decision to implant an Impella or vaECMO was based on operator's clinical evaluation (which was based on individual patients' parameters, including severity of shock, prior resuscitation, bleeding risk, isolated left ventricular failure, biventricular failure, combined cardiorespiratory failure). In brief, the Impella device is our first choice of MCS in patients with CS due to isolated left ventricular (LV) failure. In cases with biventricular failure or cases with combined LV and pulmonary failure we implant a vaECMO first. If vaECMO leads to LV distension and worsening pulmonary edema, an Impella device for venting is additionally inserted. The development of respiratory failure, right heart failure, hemodynamic deterioration, progressive multiorgan failure while on Impella support is, according to our institutional algorithm, an indication for additional implantation of vaECMO. Besides mechanical circulatory support, all patients received standardized medical care and management in accordance with guidelines. If patients received ultimately both devices, they were analyzed according to the device first implanted.

The investigation conforms with the principles outlined in the Declaration of Helsinki. The study was approved by the local ethics committee of the Philipps University of
Marburg. The need for informed consent was waived due to the retrospective nature of the study.

\section{Patients' management}

All MCS devices were implanted percutaneously in the catheterization laboratory by experienced operators. The Impella pump (Abiomed) was implanted through the femoral artery and placed via the retrograde approach through the aortic valve into the left ventricle under fluoroscopic control. The vaECMO (Maquet Getinge Group) was inserted percutaneously using arterial $(17 \mathrm{~F})$ and venous $(21 \mathrm{~F}$ for female and 23F for male) femoral cannulas with an additional antegrade femoral limb perfusion cannula. All cardiac arrest patients were treated with targeted temperature management (mild hypothermia of $34^{\circ} \mathrm{C}$ ) for $24 \mathrm{~h}$ with an endovascular cooling device (Thermogard Temperature Management System, Zoll). Inotropes and vasopressors were used to obtain a mean arterial pressure $\geq 65 \mathrm{mmHg}$. Circulatory support flow was adjusted to maintain mean arterial pressure $\geq 65 \mathrm{mmHg}$ with the lowest possible dose of catecholamines and to cover metabolic needs as assessed by central venous oxygen saturation $(\geq 70 \%)$ and serum lactate levels $(<2.0 \mathrm{mmol} / \mathrm{L})$. The decision to wean the circulatory support device was based on resolution of shock and clinical assessment. Weaning process was performed by gradually decreasing support. Once the support of the device was reduced to low levels (for Impella performance level 1 and for vaECMO < $1.5 \mathrm{~L} / \mathrm{min}$ ) with stable mean arterial pressure $\geq 65 \mathrm{mmHg}$, no or low doses of catecholamines, central venous oxygen saturation $\geq 70 \%$ and serum lactate levels $<2.0 \mathrm{mmol} / \mathrm{L}$ the device was removed in ICU and hemostasis was achieved with mechanical compression (St. Jude Medical FemoStop).

\section{Data collection and outcome variables}

Intrahospital data, outcomes and follow-up data were collected from the medical charts. Our primary outcomes were survival to hospital discharge and at 6-month. Secondary endpoints were complications. Complications included device-related vascular complications (bleeding at access-site requiring transfusion, limb ischemia requiring removal of the device, surgery or interventional repair), myocardial reinfarction, stroke and other nondevice-related bleeding. Access-site bleeding requiring transfusion was defined as bleeding at the cannulation site with need of transfusion of at least two red blood cell (RBC) units. Cerebral functional status in cardiac arrest patients was determined according to the Pittsburgh cerebral performance category (CPC) based on medical records or discharge summary abstracts. 


\section{Statistical analysis}

All data were analyzed retrospectively. Data are presented as absolute variables and percentages (\%) for categorical variables and either median with interquartile range [IQR 25th-75th percentile] or mean with standard deviation according to the distribution of the variables. We assessed normality using Shapiro-Wilk test as well as Pearson Tests. After testing for normal distribution, Student's t test or Mann-Whitney test was implemented to test for differences between the various characteristics. For categorical variables Fisher's exact test or Chi-square test were used, as appropriate. Interactions between nominal variables were measured with lambda coefficient. Patients at risk were assessed with the log-rank test of the survival analysis. The variables were dichotomized according to median in overall population, when they were not linearly distributed. An initial analysis was performed in order to identify the variables associated with outcome mortality in the overall population. A separate analysis was performed in order to identify the variables with a different distribution among the groups of devices. All these variables are presented in the Table 1. Age, Charlson Comorbidity Index (CCI), vasoactive score, creatinine, GFR, $\mathrm{pH}, \mathrm{PaO}_{2} / \mathrm{FiO}_{2}$, etiology of cardiogenic shock and prior CPR were included in the model as significantly associated with outcome in univariate analysis or as clinically meaningful. Propensity score matching was used to balance observed covariates in treatment groups $[4,5]$. In this study, the propensity score was the conditional probability for getting vaECMO for $\mathrm{CS}$, as a binary dependent variable, under a set of measurements. Age, CCI, vasoactive score, creatinine, $\mathrm{pH}$, etiology of shock, $\mathrm{PaO}_{2} / \mathrm{FiO}_{2}$ and prior $\mathrm{CPR}$ were added into a multivariable logistic regression model. The predicted probability derived from the logistic equation was used as the propensity score for each individual. The model revealed a c-statistic of 0.88 (C.I. 0.81-0.95). Then we performed a conditional logistic regression after matching on the propensity score in a 1:1 in order to identify the matched pairs. Given the frequency of the outcome in total population, our power calculation showed that we would need a minimum of 75 pairs in order to establish our superiority or non-inferiority hypothesis with a non-inferiority or superiority margin of -0.1 with a power of 0.8 and type $\alpha$ error of 0.05 . All analyses were considered statistically significant for $p<0.05$. All analyses were two-sided. Statistical analysis was performed using SPSS 24 and Graphpad Prim 6.0.

\section{Results}

\section{Patients}

From September 2014 to September 2019, a total of 423 patients with CS were treated with MCS. Among them, 300 patients (71\%) underwent Impella implantation and 123 patients $(29 \%)$ were supported with vaECMO. Within the Impella group, 227 patients (75.7\%) were supported with an Impella 2.5 and 73 (24.3\%) patients with Impella CP. Forty-two $(9.9 \%)$ patients were ultimately supported by both devices simultaneously (20 patients Impella first and 22 patients vaECMO first).

Demographic and baseline characteristics of patients are presented in Table 1. On admission, the groups were similar regarding gender distribution, cardiovascular risk factors, etiology of CS, mean blood pressure, LVEF, inotropic/vasopressor therapy and creatinine values. Compared to patients in the Impella group, patients in the vaECMO group were younger $(68.96 \pm 11.56$ versus $61.25 \pm 10.4, p<0.001)$ and presented more often with cardiac arrest $(58.9 \%$ versus $39 \%$, $p=0.0004$ ) (Table 1). Also, vaECMO supported patients had significantly worse $\mathrm{pH}(7.27 \pm 0.17$ versus $7.21 \pm 0.19$, $p=0.001)$ and significantly higher lactate levels $(6.5 \pm 4.7$ versus $9.18 \pm 5.63 \mathrm{mmol} / \mathrm{l}, p<0.001)$. Using propensity score, 83 pairs of patients were matched. The characteristics of the propensity-matched cohort were well balanced and evenly distributed regarding covariates (Table 1). The procedural characteristics of the vaECMO and Impella supported patients (overall and matched cohort) are displayed in Table 2.

\section{Outcome}

Information on hospital and 6-month mortality were available for all patients. Hospital survival rates were $47.7 \%$ in the Impella group and $37.3 \%$ in the vaECMO group $(p=0.07)$ (Table 3). Six-month survival rates were $45.7 \%$ and $35.8 \%$ $(p=0.08)$ for the Impella and vaECMO group, respectively. In the matched cohort, the hospital and 6-month survival rates were also comparable for the Impella and vaECMO group (hospital survival: $50.6 \%$ versus $38.6 \%, p=0.16$ and 6-month survival: $45.8 \%$ versus $38.6 \%, p=0.43$ ) (Fig. 1 and Table 3). Furthermore, survival rates were comparable across all tested subgroups except for patients with prior cardiac arrest where Impella support improved the primary endpoint of hospital survival (Fig. 2). Patients with prepercutaneous coronary intervention (PCI) Impella $(n=94)$ had better survival rates than patients with post-PCI Impella $(n=151)$ implantation (hospital survival and 6-month survival: $63.8 \%$ versus $51 \%, p=0.03$ ).

Regarding complications, similar rates of myocardial reinfarction (Impella: $1.3 \%$ versus vaECMO: $1.6 \%, p=0.1$ ) and stroke (Impella: $1.7 \%$ versus vaECMO: $2.4 \%, p=0.7$ ) were observed (Table 3). Device-related access-site vascular complications occurred more frequently in the vaECMO group than in the Impella group. Access-site bleedings rates requiring transfusion were significantly higher within the vaECMO group (17\% versus $7.3 \%, p=0.004)$. Vascular 
Table 1 Demographics and baseline characteristics

\begin{tabular}{|c|c|c|c|c|c|c|}
\hline \multirow[t]{2}{*}{ Variable } & \multicolumn{3}{|l|}{ Overall cohort } & \multicolumn{3}{|l|}{ Matched cohort } \\
\hline & $\begin{array}{l}\text { Impella } \\
(n=300)\end{array}$ & $\begin{array}{l}\text { vaECMO } \\
(n=123)\end{array}$ & $p$ value & $\begin{array}{l}\text { Impella } \\
(n=83)\end{array}$ & $\begin{array}{l}\text { vaECMO } \\
(n=83)\end{array}$ & $p$ value \\
\hline Age (years) & $68.96 \pm 11.56$ & $61.25 \pm 10.40$ & $<0.001$ & $63.71 \pm 11.91$ & $62.82 \pm 10.73$ & 0.61 \\
\hline Male, $n(\%)$ & $229(76.3)$ & $101(78.2)$ & 0.24 & $65(78.3)$ & $66(79.5)$ & 1 \\
\hline BMI $\left(\mathrm{kg} / \mathrm{m}^{2}\right)$ & $28.56 \pm 5.01$ & $28.42 \pm 3.57$ & 0.79 & $29.71 \pm 5.3$ & $28.84 \pm 3.5$ & 0.21 \\
\hline Baseline LVEF (\%) & $35.55 \pm 4.26$ & $34.7 \pm 4.53$ & 0.07 & $34.88 \pm 4.55$ & $34.45 \pm 4.8$ & 0.56 \\
\hline Etiology of cardiogenic shock & & & 0.23 & & & 1 \\
\hline DCM/myocarditis & $38(12.6)$ & $21(17)$ & & $12(14.4)$ & $12(14.4)$ & \\
\hline AMI & $262(87.4)$ & $106(83)$ & & $71(85.6)$ & $71(85.6)$ & \\
\hline \multicolumn{7}{|l|}{ Impella } \\
\hline 2.5 & $227(75.7)$ & - & & $66(79.5)$ & - & \\
\hline $\mathrm{CP}$ & $73(24.3)$ & & & $17(20.5)$ & & \\
\hline STEMI on presentation, $n(\%)$ & $139(46.3)$ & $60(48.7)$ & 0.67 & $34(40.9)$ & $38(45.8)$ & 0.64 \\
\hline Mechanical ventilation on admission, $n(\%)$ & $236(78.7)$ & $101(82.1)$ & 0.2 & $65(78.3)$ & $65(78.3)$ & 1 \\
\hline Prior cardiac arrest, $n(\%)$ & $117(39)$ & $72(58.1)$ & 0.0004 & $38(45.7)$ & $40(48.2)$ & 0.88 \\
\hline $\mathrm{OHCA}, n(\%)$ & $77(25.7)$ & $50(40.6)$ & & $29(34.9)$ & $30(36.1)$ & \\
\hline IHCA, $n(\%)$ & $40(35.3)$ & $22(17.9)$ & & $9(10.8)$ & $10(12)$ & \\
\hline \multicolumn{7}{|l|}{ Medical comorbidities } \\
\hline Hypertension, $n(\%)$ & $192(64)$ & $85(69.1)$ & 0.83 & $50(60.2)$ & $62(74.5)$ & 0.07 \\
\hline Diabetes, $n(\%)$ & $85(28.3)$ & $41(33.3)$ & 0.35 & $29(35)$ & $31(37.3)$ & 0.31 \\
\hline $\mathrm{PAD}, n(\%)$ & $58(19.3)$ & $21(17)$ & 0.42 & $16(19.3)$ & $11(13.3)$ & 0.4 \\
\hline Prior CAD, $n(\%)$ & $129(43)$ & $47(38.2)$ & 0.39 & $40(48.2)$ & $33(40)$ & 0.35 \\
\hline Stroke, $n(\%)$ & $32(10.7)$ & $19(15.4)$ & 0.19 & $14(16.9)$ & $11(13.3)$ & 0.67 \\
\hline Prior PCI, $n(\%)$ & $93(31)$ & $34(27.6)$ & 0.56 & $28(33.7)$ & $26(31.3)$ & 0.87 \\
\hline $\mathrm{CABG}, n(\%)$ & $35(11.7)$ & $13(10.6)$ & 0.87 & $10(12)$ & $9(10.8)$ & 1 \\
\hline Prior HF, $n(\%)$ & $42(14)$ & $16(13)$ & 0.88 & $10(12)$ & $12(14.5)$ & 0.82 \\
\hline Prior MI, $n(\%)$ & $70(23.3)$ & $27(22)$ & 0.8 & $24(28.9)$ & $23(27.7)$ & 1 \\
\hline Charlson comorbidity index & $4.66 \pm 2.32$ & $3.98 \pm 2.4$ & 0.007 & $4[3,5]$ & $4[3,5]$ & 1 \\
\hline \multicolumn{7}{|l|}{ Catecholamines } \\
\hline Vasopressors or inotropes, $n(\%)$ & $300,(100)$ & $123,(100)$ & 1 & $83(100)$ & $83(100)$ & 1 \\
\hline Vasoactive Score & $51.15[18.89,86.9]$ & $54.51[30,81.22]$ & 0.38 & $49.28[19.75,87.16]$ & $51[30,78]$ & 0.64 \\
\hline \multicolumn{7}{|l|}{ Hemodynamic variables on admission } \\
\hline Heart rate (bpm) & $94 \pm 20.6$ & $94.6 \pm 19.67$ & 0.78 & $98.16 \pm 20.71$ & $95.88 \pm 22.49$ & 0.5 \\
\hline Systolic blood pressure (mmHg) & $106.1 \pm 30.93$ & $101.5 \pm 22.67$ & .014 & $108 \pm 35.18$ & $101.3 \pm 24.53$ & 0.06 \\
\hline Diastolic blood pressure $(\mathrm{mmHg})$ & $64.13 \pm 15.73$ & $61.34 \pm 12.55$ & 0.08 & $64.71 \pm 17.39$ & $60.93 \pm 12.09$ & 0.11 \\
\hline Mean blood pressure $(\mathrm{mmHg})$ & $78.1 \pm 19.6$ & $74.71 \pm 14.44$ & 0.08 & $80.04 \pm 22.11$ & $74.37 \pm 14.91$ & 0.06 \\
\hline \multicolumn{7}{|l|}{ Blood values on admission } \\
\hline Lactate $(\mathrm{mmol} / \mathrm{l})$ & $6.5 \pm 4.7$ & $9.18 \pm 5.63$ & $<0.001$ & $6.82 \pm 5.04$ & $7.97 \pm 4.8$ & 0.14 \\
\hline GFR (ml/min) & $51[38.48,63.75]$ & $47[33,63]$ & 0.11 & $51[38,68]$ & $48.5[33.5,63]$ & 0.23 \\
\hline Arterial $\mathrm{pH}$ & $7.27 \pm 0.17$ & $7.21 \pm 0.19$ & 0.001 & $7.25 \pm 0.19$ & $7.26 \pm 0.18$ & 0.84 \\
\hline $\mathrm{PaO}_{2} / \mathrm{FiO}_{2}$ & $232[175,327]$ & $226[155,306]$ & 0.23 & $233[172,306]$ & $222[147,273]$ & 0.31 \\
\hline Creatinine (mg/dl) & $1.51[1,1.73]$ & $1.47[1.3,1.94]$ & 0.55 & $1.45[1.1,1.68]$ & $1.4[1.1,1.85]$ & 0.82 \\
\hline Hemoglobin(g/dl) & $126[120,129]$ & $123[113,140]$ & 0.1 & $123[116,139]$ & $123[115,140]$ & 0.74 \\
\hline Sodium (mmol/l) & $137 \pm 4.2$ & $137 \pm 4.51$ & 0.23 & $136.6 \pm 5.17$ & $137 \pm 4.8$ & 0.47 \\
\hline Potassium (mmol/l) & $4.2[3.9,4.3]$ & $4.1[3.9,4.4]$ & 0.08 & $4.2[3.9,4.23]$ & $4.1[3.9,4.4]$ & 0.63 \\
\hline Thrombocytes (G/l) & $219[190,262]$ & $234[157,709]$ & 0.45 & $217[172,262]$ & $240[158,709]$ & 0.81 \\
\hline
\end{tabular}

$B M I$ body mass index, $L V E F$ left ventricular ejection fraction, DCM dilated cardiomyopathy, $A M I$ acute myocardial infarction, STEMI ST elevation myocardial infarction, $O H C A$ out-of-hospital cardiac arrest, $I H C A$, intrahospital cardiac arrest, $P A D$ peripheral artery disease, $C A D$ coronary artery disease, $P C I$ percutaneous coronary intervention, $C A B G$ coronary artery bypass-graft, $H F$ heart failure, $M I$ myocardial infarction, $G F R$ glomerular filtration rate; Numbers are presented as mean ( \pm standard deviation), median [interquartile range, IQR 25 th-75th percentile] or frequency (percentile) 
Table 2 Procedural characteristics of the overall and matched cohort

\begin{tabular}{|c|c|c|c|c|c|c|}
\hline \multirow[t]{2}{*}{ Variable } & \multicolumn{3}{|l|}{ Overall cohort } & \multicolumn{3}{|l|}{ Matched cohort } \\
\hline & $\begin{array}{l}\text { Impella } \\
(n=300)\end{array}$ & $\begin{array}{l}\text { vaECMO } \\
(n=123)\end{array}$ & $p$ value & $\begin{array}{l}\text { Impella } \\
(n=83)\end{array}$ & $\begin{array}{l}\text { vaECMO } \\
(n=83)\end{array}$ & $p$ value \\
\hline Door to MCS (min) & $356.6 \pm 87.81$ & $350.4 \pm 84.21$ & 0.76 & $353.3 \pm 84.18$ & $342.6 \pm 85.9$ & 0.42 \\
\hline Duration of support (hours) & $128.3 \pm 37.21$ & $128.3 \pm 43.78$ & 0.04 & $118.8 \pm 36$ & $130.2 \pm 47.2$ & 0.76 \\
\hline Culprit vessel, $n(\%)^{\mathrm{a}}$ & & & NS & & & NS \\
\hline Left main & $33(12.6)$ & $6(5.7)$ & & $11(10)$ & $12(16.9)$ & \\
\hline LAD & $125(47.7)$ & $47(44.3)$ & & $28(39.4)$ & $28(39.4)$ & \\
\hline $\mathrm{LCx}$ & $44(16.8)$ & $19(17.9)$ & & $16(22.5)$ & $16(22.5)$ & \\
\hline RCA & $48(18.3)$ & $25(23.5)$ & & $10(13.7)$ & $10(92.5)$ & \\
\hline Bypass-graft & $12(4.5)$ & $9(8.5)$ & & $4(15,4)$ & $5(7.5)$ & \\
\hline Multivessel disease & $141(53.8)$ & $74(69.8)$ & 0.0051 & $42(66.7)$ & $42(64.6)$ & 1 \\
\hline Multivessel Intervention & $89(34)$ & $39(36.7)$ & 0.63 & $28(38.3)$ & $24(33.8)$ & 0.6 \\
\hline Unsuccessful PCI & $13(4.9)$ & $3(2.9)$ & 0.57 & $2(2.8)$ & $0(0)$ & 0.5 \\
\hline Contrast Agent (ml) & $255[180,320]$ & $250[180,270]$ & 0.06 & $255[200,350]$ & $250[180,270]$ & 0.004 \\
\hline
\end{tabular}

$M C S$ mechanical circulatory support, $L A D$ left coronary artery, $L C x$ left circumflex artery, $R C A$ right coronary artery, $P C I$ percutaneous coronary intervention. Numbers are presented as mean ( \pm standard deviation), median [interquartile range, IQR 25 th-75th percentile] or frequency (percentile); NS non-significant

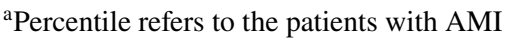

Table 3 Outcomes

\begin{tabular}{|c|c|c|c|c|c|c|}
\hline Outcome & $\begin{array}{l}\text { Impella } \\
(n=300)\end{array}$ & $\begin{array}{l}\text { vaECMO } \\
(n=123)\end{array}$ & $p$ value & $\begin{array}{l}\text { Impella-matched } \\
(n=83)\end{array}$ & $\begin{array}{l}\text { vaECMO-matched } \\
(n=83)\end{array}$ & $p$ value \\
\hline Survival to hospital discharge, $n(\%)$ & $143(47.7)$ & $46(37.3)$ & 0.07 & $42(50.6)$ & $32(38.6)$ & 0.16 \\
\hline Survival at 6-month, $n(\%)$ & $137(45.7)$ & $44(35.8)$ & 0.07 & $38(45.8)$ & $32(38.6)$ & 0.43 \\
\hline Access-site bleeding requiring transfusion, $n(\%)$ & $22(7.3)$ & $21(17)$ & 0.004 & $10(12)$ & $12(9.8)$ & 0.82 \\
\hline Limb ischemia requiring intervention, $n(\%)$ & $23(7.7)$ & $21(17)$ & 0.008 & $7(8.4)$ & $12(14.5)$ & 0.33 \\
\hline Myocardial Reinfarction, $n(\%)$ & $4(1.3)$ & $2(1.6)$ & 1 & $1(1.2)$ & $1(1.2)$ & 1 \\
\hline Stroke, $n(\%)$ & $5(1.7)$ & $3(2.4)$ & 0.7 & $2(2.4)$ & $2(2.4)$ & 1 \\
\hline Non-device-related bleeding, $n(\%)$ & $6(2)$ & $9(7.3)$ & 0.02 & $2(2.4)$ & $7(8.4)$ & 0.17 \\
\hline
\end{tabular}

Numbers are presented as mean ( \pm standard deviation), median [interquartile range, IQR 25th-75th percentile] or frequency (percentile)

access-site ischemic complications requiring removal of the device, surgery or percutaneous intervention occurred in $17 \%$ and $7.7 \%(p=0.008)$ of patients in the vaECMO and Impella group, respectively (Table 3 ).

\section{Discussion}

We here describe a large single-center, propensity-matched study of patients with CS supported with either Impella 2.5/ $\mathrm{CP}$ or vaECMO. To our knowledge, this is the largest singlecenter cohort comparing Impella and vaECMO in patients with CS. In our analysis, Impella $2.5 / \mathrm{CP}$ and vaECMO were associated with similar outcome in patients with CS. In the overall cohort, hospital and 6-month survival rates were comparable among the Impella and vaECMO group.
However, several baseline characteristics lead to consider the vaECMO group a higher-risk population over the Impella group. Patients of the vaECMO group had a higher percentage of prior cardiac arrest, higher baseline lactate levels and lower $\mathrm{pH}$. These factors are known to be associated with worse outcome in CS patients [6-8]. Therefore, since we documented significant differences in baseline characteristics especially with respect to critical prognostic parameters we performed a propensity-matching to enhance comparability between the two groups. After propensity-matching, the baseline characteristics of the matched cohort were comparable and evenly distributed between the Impella and vaECMO group. After propensity-matching, Impella and vaECMO supported patients showed again comparable hospital and 6-month survival rates. This result was consistent among all tested subgroups, in contrast to patients with 


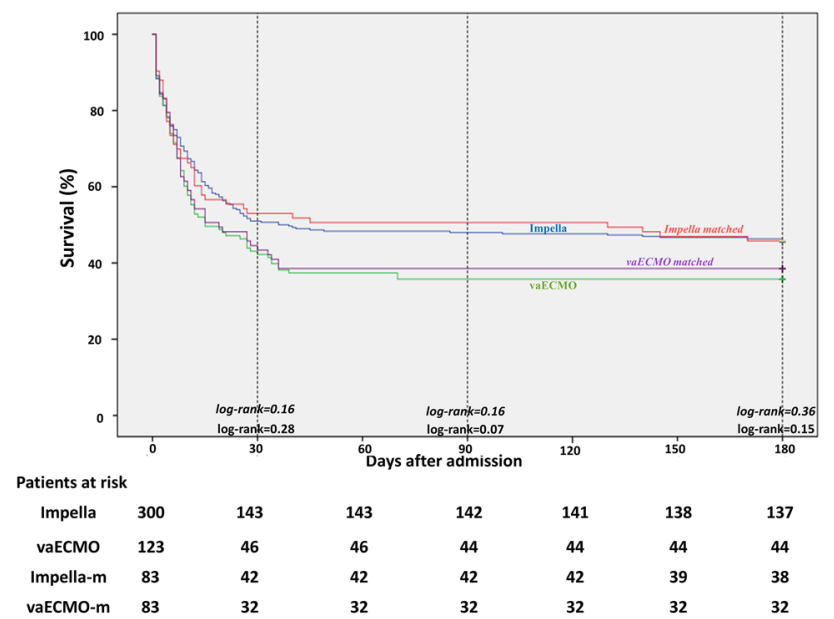

Fig. 1 Kaplan-Meier curves demonstrating 6-month survival in Impella and vaECMO patients. Impella-m Impella-matched cohort, vaECMO- $m$ vaECMO-matched cohort

prior cardiac arrest in whom Impella support was associated with improved hospital survival rates (Fig. 2). In the particular group of patients with post-cardiac arrest shock and vaECMO support high rates of access-site complications (up to $29 \%$ ) were previously reported $[9,10]$. In accordance, patients in the vaECMO group with prior cardiac arrest had, in our analysis, device-associated vascular complications in $23 \%$ of cases (versus $8.4 \%$ in Impella patients with prior cardiac arrest), which may have contributed to the result in this subgroup. However, the subgroup analysis of cardiac arrest patients should be interpreted with caution and needs further evaluation in a sufficiently powered study.

Given the persistently poor outcomes in patients with CS, interest in the role and appropriate selection of these two invasive devices has been increasing. However, the selection of type of MCS in patients with CS is still unclear and varies among institutions, since solid evidence from randomized controlled trial are, so far, lacking. Therefore, comparative analyses of real-life use of MCS in patients with CS regarding outcomes and complications as presented in our investigation are important and add information to the current knowledge. To date, very few investigations compared outcomes of patients treated with these two MCS devices [11-14] and no randomized data are available favoring one type of device over the other. Recently, Garan and colleagues, prospectively compared outcomes of Impella and vaECMO in 51 patients with acute myocardial infarction (AMI)-related CS [13]. In accordance with our results, their data demonstrated that the two devices were associated with similar outcomes [13]. Karami and colleagues conducted a retrospective study of CS patients requiring Impella or vaECMO [14]. In this study, Impella and vaECMO support was again not associated with a difference in 30-day mortality. Furthermore, in another retrospective analysis by Schiller and colleagues, short and long-term survival was not measurably different between Impella and vaECMO supported patients with CS, even after adjustment of disease severity through the SAVE score [11].

Randomized trials adequately powered are particularly challenging to conduct in patients with CS. Several

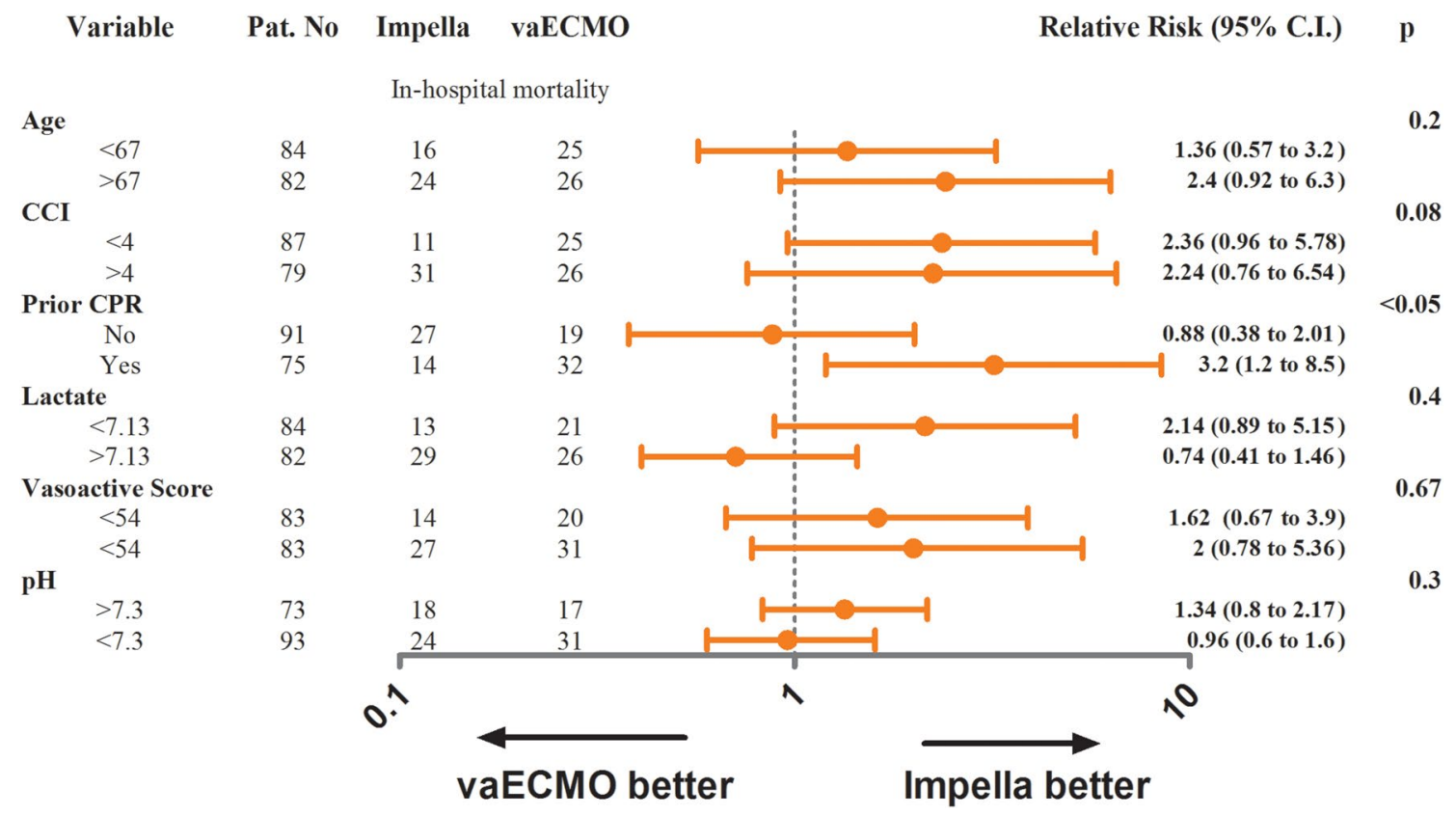

Fig. 2 Forest plot displaying the relative risk of different tested subgroups for the primary endpoint hospital mortality in matched cohort 
randomized trials investigating MCS in CS, like the DanGer SHOCK (NCT01633502), ECLS-SHOCK (NCT03637205) and EUROSHOCK (NCT03813134) trial, are ongoing and their results awaited. However, these trials compare either vaECMO or Impella to standard medical treatment. The lack of an appreciably and clear benefit of one device over the other, warrants, in our view, also a randomized controlled trial comparing head-to-head these two devices in patients with CS.

Regarding safety outcomes, we found no differences in stroke or myocardial reinfarction between Impella and vaECMO supported patients. However, as expected access-site complications occurred more frequently in the vaECMO group than in the Impella group. This finding is in line with previous investigations [14-16]. Recently, a large-scale propensity-matched, registry-based retrospective cohort study observational including 1768 patients with CS treated with Impella reported high rates of major bleeding of 31.3\% [17], which was markedly higher compared to the bleeding rates in our Impella cohort. On the other side, further retrospective studies reported lower vascular complication rates comparable to our results $[15,16]$. Registry data including 112 patients supported with Impella for AMI-related CS reported an overall vascular complication rate of $17 \%$ [15]. Limb ischemia occurred in 3.5\% and major access-site bleeding in $9.8 \%$ of patients [15]. Another retrospective analysis of 237 patients with AMI-associated CS treated with Impella reported peripheral ischemic vascular complications in 9.8\% of Impella patients [16]. In patients treated with vaECMO vascular complications occur at a high rate as repeatedly reported in several investigations. A meta-analysis of 1866 patients supported with vaECMO for CS reported major bleeding rates of $40.8 \%$ and a lower extremity ischemia rate of $16.9 \%$ [18], whereas the study by Combes and colleagues reported femoral bleeding in $32 \%$ and peripheral leg ischemia in $20 \%$ of vaECMO patients [19]. The higher rates of peripheral vascular complications in the vaECMO group as compared to the Impella group might be attributed to the larger vascular access needed for vaECMO implantation.

\section{Limitations}

Even though this might be the largest comparison of vaECMO versus Impella, this study has several limitations. First, our observations are obviously limited by the retrospective and non-randomized design of our study and therefore, prone to selection bias. Second, our investigation was a single-center study, but based on otherwise standardized procedures. Third, the device selection was based on operator's discretion and our institutional algorithm and not directed by a study protocol.
But beside some limitations, the strength of this matched analysis is that it is, so far, the largest single-center investigation comparing CS patients treated with either Impella 2.5/ $\mathrm{CP}$ or vaECMO.

Thus, since our results are preliminary, the association between these two MCS devices and outcomes of patients with CS warrants further evaluation in randomized studies.

\section{Conclusions}

In this retrospective analysis of a large cohort of patients with CS, treatment with Impella $2.5 / \mathrm{CP}$ or vaECMO was associated with similar hospital and 6-month survival rates. Device-related access-site vascular complications occurred more frequently in patients with vaECMO support than in Impella patients. A randomized trial is warranted to examine the effects of these MCS devices on outcomes and to determine the optimal device choice in patients with CS.

Funding Open Access funding enabled and organized by Projekt DEAL.

\section{Compliance with ethical standards}

Conflict of interest Dr. Schieffer received speaker's honoraria from Maquet Getinge Group, Novartis, Edwards and Abiomed. Drs. Karatolios, Markus, Luesebrink and Jerrentrup have received speaker's honoraria from Abiomed. Drs. Chatzis, Syntila, Ahrens and Divchev have no disclosures.

Open Access This article is licensed under a Creative Commons Attribution 4.0 International License, which permits use, sharing, adaptation, distribution and reproduction in any medium or format, as long as you give appropriate credit to the original author(s) and the source, provide a link to the Creative Commons licence, and indicate if changes were made. The images or other third party material in this article are included in the article's Creative Commons licence, unless indicated otherwise in a credit line to the material. If material is not included in the article's Creative Commons licence and your intended use is not permitted by statutory regulation or exceeds the permitted use, you will need to obtain permission directly from the copyright holder. To view a copy of this licence, visit http://creativecommons.org/licenses/by/4.0/.

\section{References}

1. Stretch R, Sauer CM, Yuh DD, Bonde P (2014) National trends in the utilization of short-term mechanical circulatory support: incidence, outcomes, and cost analysis. J Am Coll Cardiol 64:14071415. https://doi.org/10.1016/j.jacc.2014.07.958

2. Rihal CS, Naidu SS, Givertz MM et al (2015) 2015 SCAI/ACC/ HFSA/STS clinical expert consensus statement on the use of percutaneous mechanical circulatory support devices in cardiovascular care: endorsed by the American Heart Assocation, the Cardiological Society of India, and Sociedad Latino Americana de 
Cardiologia Intervencion; Affirmation of Value by the Canadian Association of Interventional Cardiology-Association Canadienne de Cardiologie d'intervention. J Am Coll Cardiol 65:e7-e26

3. Burkhoff D, Sayer G, Doshi D, Uriel N (2015) Hemodynamics of mechanical circulatory support. J Am Coll Cardiol 66:2663-2674. https://doi.org/10.1016/j.jacc.2015.10.017

4. Austin PC (2011) An introduction to propensity score methods for reducing the effects of confounding in observational studies. Multivariate Behav Res 46:399-424. https://doi.org/10.1080/00273 171.2011.568786

5. Chen Y-S, Lin J-W, Yu H-Y, Ko W-J, Jerng J-S, Chang W-T, Chen W-J, Huang S-C, Chi N-H, Wang C-H, Chen L-C, Tsai P-R, Wang S-S, Hwang J-J, Lin F-Y (2008) Cardiopulmonary resuscitation with assisted extracorporeal life-support versus conventional cardiopulmonary resuscitation in adults with in-hospital cardiac arrest: an observational study and propensity analysis. Lancet 372:554-561. https://doi.org/10.1016/S0140-6736(08)60958-7

6. Acharya D (2018) Predictors of outcomes in myocardial infarction and cardiogenic shock. Cardiol Rev 26:255-266. https://doi. org/10.1097/CRD.0000000000000190

7. Harjola V-P, Lassus J, Sionis A, Køber L, Tarvasmäki T, Spinar J, Parissis J, Banaszewski M, Silva-Cardoso J, Carubelli V, Di Somma S, Tolppanen H, Zeymer U, Thiele H, Nieminen MS, Mebazaa A (2015) Clinical picture and risk prediction of shortterm mortality in cardiogenic shock. Eur J Heart Fail 17:501-509. https://doi.org/10.1002/ejhf.260

8. Vergara R, Valenti R, Migliorini A, Cerisano G, Carrabba N, Giurlani L, Antoniucci D (2017) A new risk score to predict longterm cardiac mortality in patients with acute myocardial infarction complicated by cardiogenic shock and treated with primary percutaneous intervention. Am J Cardiol 119:351-354. https:// doi.org/10.1016/j.amjcard.2016.10.034

9. de Chambrun MP, Bréchot N, Lebreton G, Schmidt M, Hekimian G, Demondion P, Trouillet J-L, Leprince P, Chastre J, Combes A, Luyt C-E (2016) Venoarterial extracorporeal membrane oxygenation for refractory cardiogenic shock post-cardiac arrest. Intensive Care Med 42:1999-2007. https://doi.org/10.1007/s0013 4-016-4541-y

10. Bougouin W, Aissaoui N, Combes A, Deye N, Lamhaut L, Jost D, Maupain C, Beganton F, Bouglé A, Karam N, Dumas F, Marijon E, Jouven X, Cariou A (2016) Post-cardiac arrest shock treated with veno-arterial extracorporeal membrane oxygenation: an observational study and propensity-score analysis. Resuscitation 110:126-132. https://doi.org/10.1016/j.resuscitation.2016.11.005

11. Schiller P, Hellgren L, Vikholm P (2018) Survival after refractory cardiogenic shock is comparable in patients with Impella and veno-arterial extracorporeal membrane oxygenation when adjusted for SAVE score. Eur Heart J Acute Cardiovasc Care 8:329-337. https://doi.org/10.1177/2048872618799745

12. Mourad M, Gaudard P, La Arena DP, Eliet J, Zeroual N, Rouvière P, Roubille F, Albat B, Colson PH (2017) Circulatory support with extracorporeal membrane oxygenation and/or Impella for cardiogenic shock during myocardial infarction. ASAIO J 64:708714. https://doi.org/10.1097/MAT.0000000000000704

13. Garan AR, Takeda K, Salna M, Vandenberge J, Doshi D, Karmpaliotis D, Kirtane AJ, Takayama H, Kurlansky P (2019) Prospective comparison of a percutaneous ventricular assist device and venoarterial extracorporeal membrane oxygenation for patients with cardiogenic shock following acute myocardial infarction. J Am Heart Assoc 8:e012171. https://doi.org/10.1161/ JAHA.119.012171

14. Karami M, Uil Den CA, Ouweneel DM, Scholte NT, Engström AE, Akin S, Lagrand WK, Vlaar AP, Jewbali LS, Henriques JP (2019) Mechanical circulatory support in cardiogenic shock from acute myocardial infarction: Impella CP/5.0 versus ECMO. Eur Heart J Acute Cardiovasc Care 9:164-172. https://doi. org/10.1177/2048872619865891

15. Ouweneel DM, de Brabander J, Karami M, Sjauw KD, Engström AE, Vis MM, Wykrzykowska JJ, Beijk MA, Koch KT, Baan J, de Winter RJ, Piek JJ, Lagrand WK, Cherpanath TG, Driessen AH, Cocchieri R, de Mol BA, Tijssen JG, Henriques JP (2018) Real-life use of left ventricular circulatory support with Impella in cardiogenic shock after acute myocardial infarction: 12 years AMC experience. Eur Heart J Acute Cardiovasc Care 8:338-349. https://doi.org/10.1177/2048872618805486

16. Schrage B, Ibrahim K, Loehn T, Werner N, Sinning J-M, Pappalardo F, Pieri M, Skurk C, Lauten A, Landmesser U, Westenfeld R, Horn P, Pauschinger M, Eckner D, Twerenbold R, Nordbeck P, Salinger T, Abel P, Empen K, Busch MC, Felix SB, Sieweke J-T, Møller JE, Pareek N, Hill J, MacCarthy P, Bergmann MW, Henriques JPS, Möbius-Winkler S, Schulze PC, Ouarrak T, Zeymer U, Schneider S, Blankenberg S, Thiele H, Schäfer A, Westermann D (2019) Impella support for acute myocardial infarction complicated by cardiogenic shock. Circulation 139:1249-1258. https:// doi.org/10.1161/CIRCULATIONAHA.118.036614

17. Dhruva SS, Ross JS, Mortazavi BJ, Hurley NC, Krumholz HM, Curtis JP, Berkowitz A, Masoudi FA, Messenger JC, Parzynski CS, Ngufor C, Girotra S, Amin AP, Shah ND, Desai NR (2020) Association of use of an intravascular microaxial left ventricular assist device vs intra-aortic balloon pump with in-hospital mortality and major bleeding among patients with acute myocardial infarction complicated by cardiogenic shock. JAMA 323:734. https://doi.org/10.1001/jama.2020.0254

18. Cheng R, Hachamovitch R, Kittleson M, Patel J, Francisko A, Moriguchi J, Esmailian F, Azarbal B (2014) Complications of extracorporeal membrane oxygenation for treatment of cardiogenic shock and cardiac arrest: a meta-analysis of 1,866 adult patients. Ann Thorac Surg 97:610-616. https://doi.org/10.1016/j. athoracsur.2013.09.008

19. Combes A, Leprince P, Luyt C-E, Bonnet N, Trouillet J-L, Léger P, Pavie A, Chastre J (2008) Outcomes and long-term quality-oflife of patients supported by extracorporeal membrane oxygenation for refractory cardiogenic shock. Crit Care Med 36:14041411. https://doi.org/10.1097/CCM.0b013e31816f7cf7 